\title{
Concepções de ingressantes da Moradia Estudantil sobre as disciplinas Cálculo
}

\author{
Concepciones de ingreso de la Vivienda Estudiantil sobre las disciplinas
}

\author{
Cálculo
}

\section{Concepções de Ingressantes da Moradia Estudantil sobre disciplinas}

\author{
Cálculo
}

\author{
Joice Rejane Pardo Maurell ${ }^{1}$ \\ Celiane Costa Machado
}

\begin{abstract}
Resumo
Este texto se insere em uma pesquisa maior, que culminará em uma tese de doutorado, pelo Programa de PósGraduação em Educação em Ciências: química da vida e saúde da Universidade Federal do Rio Grande - FURG. A referida pesquisa tem o objetivo de compreender como os sujeitos ingressantes da moradia estudantil desenvolvem estratégias para aprender e aprovar nas disciplinas Cálculo. Contudo, nesse texto, buscando uma primeira aproximação com os sujeitos da pesquisa, metodologicamente, optou-se por fazer o mapa do campo, dividido em dois movimentos e, levantar alguns dados para a análise. O objetivo inicial foi conhecer o contexto, a fim de traçar o perfil dos 24 estudantes que ingressaram na moradia estudantil na FURG, no primeiro semestre de 2017 e estavam matriculados nas disciplinas Cálculo. No primeiro movimento foram levantados dados que antecedem o ingresso na Universidade, como: proveniência regional, gênero, idade e rede de Educação Básica e, posteriormente, dados acadêmicos, como: modalidade de ingresso pelo Sistema de Seleção Unificada - SISU, curso, a disciplina Cálculo em que estavam matriculados e suas respectivas ementas. Traçado o perfil dos sujeitos realizou-se um segundo movimento, no qual foram entrevistados os 11 primeiros sujeitos da pesquisa. Nas entrevistas os estudantes definiram a disciplina em uma palavra e relataram as suas estratégias de estudos, avaliando-as. Contudo, o presente trabalho está se detendo em discutir as respostas referentes ao questionamento: "Defina a disciplina cálculo em uma palavra.". Como resposta ao questionamento emergiram expressões como: divisor de águas, paixão, medo, difícil, possibilidades, empecilho e necessário.
\end{abstract}

Palavras-Chave: Perfil, Ingressantes, Moradia Estudantil, Cálculo, Concepções.

\section{Resumen}

Este texto se inserta en una investigación más grande, que culminará en una tesis de doctorado, por el Programa de Postgrado en Educación en Ciencias: química de la vida y salud de la Universidad Federal de Rio Grande - FURG. La referida investigación tiene el objetivo de comprender cómo los sujetos ingresantes de la vivienda estudiantil desarrollan estrategias para aprender y aprobar en las disciplinas Cálculo. Sin embargo, en ese texto, buscando una primera aproximación con los sujetos de la investigación, metodológicamente, se optó por hacer el mapa del campo, dividido en dos movimientos y, levantar algunos datos para el análisis. El objetivo inicial fue conocer el contexto, a fin de trazar el perfil de los 24 estudiantes que ingresaron en la

\footnotetext{
1 "Doutora em Matemática Aplicada; Universidade Federal do Rio Grande - FURG; Rio Grande, Rio Grande do Sul e Brasil; celianemachado@ furg.br".

2 "Mestre em Educação Ambiental; Universidade Federal do Rio Grande - FURG; Rio Grande, Rio Grande do Sul e Brasil; joicerejane@furg.br".

Trabalho apresentado no III Encontro Humanístico Multidisciplinar (EHM) e do II Congresso Latino-Americano de Estudos Humanísticos Multidisciplinares, sob a temática "Interlocuções com o contexto Sociopolítico Atual”, que será realizado nas dependências da Universidade Federal do Pampa, na cidade de Jaguarão, Rio Grande do Sul, Brasil, de 07 à 09 de novembro de 2017.
} 
vivienda estudiantil en la FURG, en el primer semestre de 2017 y estaban matriculados en las disciplinas Cálculo. En el primer movimiento, se levantaron datos que anteceden al ingreso en la Universidad, como: proveniencia regional, género, edad y red de Educación Básica y, posteriormente, datos académicos, como: modalidad de ingreso por el Sistema de Selección Unificada - SISU, curso, a disciplina Cálculo en que estaban matriculados y sus respectivas menús. Trazado el perfil de los sujetos, fue realizado un segundo movimiento, en el cual fueron entrevistados los 11 primeros sujetos de la investigación. En las entrevistas los estudiantes definieron la disciplina en una palabra y relataron sus estrategias de estudios, evaluándolas. Sin embargo, el presente trabajo se está deteniendo en discutir las respuestas referentes al cuestionamiento: "Defina la disciplina de cálculo en una palabra.". Como respuesta al cuestionamiento surgieron expresiones como: divisor de aguas, pasión, miedo, difícil, posibilidades, impedimento y necesario.

Palabras claves: Perfil, Ingresos, Vivienda Estudiantil, Cálculo, Concepciones.

\begin{abstract}
This text is part of a larger research, culminating in a PhD thesis, by the Graduate Program in Science Education: Life and Health Chemistry of the Federal University of Rio Grande - FURG. The aim of this research is to understand how students entering the student residence develop strategies to learn and approve in the subjects Calculus. However, in this text, seeking a first approximation with the subjects of the research, methodologically, it was decided to make the map of the field, divided in two movements and, to collect some data for the analysis. The initial objective was to know the context in order to trace the profile of the 24 students who entered the student residence at FURG in the first half of 2017 and were enrolled in the courses Calculus. In the first movement, data were collected that precede the entrance to the University, such as: regional provenance, gender, age and Basic Education network and, later, academic data, such as: Unified Selection System (SISU) discipline Calculation in which they were enrolled and their respective menus. Once the profile of the subjects was drawn, a second movement was carried out, in which the first 11 subjects of the research were interviewed. In the interviews the students defined the discipline in a word and reported on their study strategies, evaluating them. However, the present work is focusing on discussing the answers regarding the question: "Define the discipline of calculation in a word." In response to the questioning emerged expressions such as: divisor of water, passion, fear, difficult, possibilities, hindrance and necessary.
\end{abstract}

Keywords: Profile, Ingressants, Student Housing, Calculus, Conceptions.

\title{
1. Introdução
}

Nas últimas décadas, muitas pesquisas foram realizadas com temas relacionados à Educação Matemática; a diversidade de tópicos abrange todos os níveis de ensino nos aspectos relacionados ao processo de ensino e aprendizagem e a construção de saberes matemáticos. No Ensino Superior, de acordo com Malta (2004), os trabalhos convergem para as disciplinas iniciais dos cursos da área das ciências exatas, entre elas o Cálculo, devido ao número crescente de reprovações.

No que se refere a produção acadêmica, que tem como tema a moradia estudantil, Garrido e Mercuri (2013) localizaram nos periódicos nacionais 23 estudos, publicados entre 2000 e 2009, cujo resultado da análise dos objetivos das produções apontou diferentes focos, agrupados em três categorias: o estudante morador, a moradia estudantil e as ações da assistência estudantil. Entre estes trabalhos, foram produzidas cinco dissertações de mestrado, mas nenhuma tese de doutorado, nem reflexões acerca das aprendizagens em disciplinas específicas. Sobre o desempenho acadêmico, fazendo uma análise da moradia estudantil nos Estados Unidos da América, Yeung (apud GARRIDO e MERCURI, 2013) 
efetuou um estudo cuja finalidade foi a de identificar o impacto da experiência de morar em residências situadas no campus sobre o rendimento médio do estudante e sobre sua integração acadêmica e social. O tipo de residência foi categorizado como: moradia no campus, residência com os familiares e outros espaços de moradias. $\mathrm{O}$ autor concluiu que morar no campus exerce influência positiva na integração acadêmica e social do estudante, enquanto morar com os familiares exerce ligeira influência positiva sobre o desempenho acadêmico (YEUNG apud GARRIDO e MERCURI, 2013, p. 88-89).

No que tange especificamente ao contexto deste estudo, nas entrevistas realizadas pelo setor pedagógico da Pró-reitora de Assuntos Estudantis - PRAE, da Universidade Federal do Rio Grande - FURG, na qual uma das pesquisadoras atua como pedagoga educacional, os estudantes matriculados em Cálculo relatam situações que envolvem uma extrema dificuldade na disciplina, como a insegurança por ter cursado o Ensino Médio em uma escola pública, podendo ocasionar falta de conhecimentos prévios para compreender os conceitos. Relatam ainda desorganização na rotina de estudos, culminando na falta de tempo para resolver as listas de exercícios, ansiedade e lapsos de memória nas atividades de avaliação, descontrole emocional, entre outros. Conforme pesquisas realizadas na área, Silva (2011) afirma

\begin{abstract}
Ao ingressarem no curso superior, os estudantes trazem suas expectativas: Aqueles que no Ensino Médio logravam sempre boas avaliações em matemática, levam para a universidade a esperança de que o curso de Cálculo não deva representar obstáculos para o seu aprendizado. Entretanto, ao se depararem com questões globais envolvendo os temas anteriormente estudados, em geral de modo departamentalizado, acrescidas de novas ideias impactantes como o infinito, as aproximações, a continuidade, a incomensurabilidade, etc., quase sempre veem frustradas suas expectativas iniciais (p. 400).
\end{abstract}

No entanto, para Alarcão (2000),

a atribuição de culpas ao ensino básico e secundário ou aos estudantes, se bem que tenha algum fundamento, é explicação demasiado simplista para poder sequer explicar o fenômeno e, muito menos, contribuir para a sua resolução. Igualmente simplista, mas que por vezes com algum fundamento, é a atribuição de culpas à falta de competência pedagógica dos docentes do ensino superior, afirmação que hoje, infelizmente, ameaça transformar-se num chavão. (p. 14)

Ademais, as experiências no acompanhamento pedagógico desses estudantes têm mostrado que os elementos anteriormente descritos refletem diretamente no desempenho acadêmico. E ainda, a falta de identificação dos acadêmicos com a área e/ou com o curso escolhido, além da retenção nas disciplinas Cálculo, uma ou consecutivas vezes, culminando no indeferimento de benefícios como: alimentação, transporte, auxílio permanência, auxílio infância e auxílio moradia e, ainda, desgaste físico, emocional e psicológico, alguns desencadeando os processos de desligamento a pedido ou por abandono do curso. 
Entretanto, embora os estudantes sejam atendidos por demanda espontânea, encaminhamentos de professores ou coordenações e, ainda, em cursos como o Pré-Cálculo, são pontuais os acompanhamentos processuais, realizado por pedagogos educacionais, no primeiro ano do curso (SCHIRMER, BURLAMAQUI e MAURELL, 2016, p. 147 - 156). Atinente, considerando a demanda, que é crescente e o número de profissionais disponíveis para realizar o acompanhamento desses acadêmicos é emergente pensar em estratégias para qualificar o processo.

Nesse sentido, o objetivo da pesquisa aqui apresentada é compreender as concepções dos estudantes acerca das disciplinas Cálculo, após cursar o primeiro semestre do curso. Com esse objetivo, metodologicamente, optou por fazer um "mapa do campo" (BIEMBENGUT, 2008), divido em dois movimentos, cuja a organização do artigo segue a mesma proposta. O primeiro movimento do artigo apresenta o perfil dos estudantes no período anterior ao ingresso e, depois como acadêmicos ingressantes. O segundo movimento detém-se a relatar o processo de intervenção e apresentar as expressões que os 11 entrevistados utilizaram para definir as disciplinas Cálculo, as quais foram analisadas em uma abordagem qualitativa.

\section{Situando a pesquisa}

Metodologicamente, a presente pesquisa tem enfoque na abordagem qualitativa no processo de análise dos dados, mesmo que para fazer o mapa do campo, incialmente, os gráficos e tabelas apresentaram-se como mais efetivos. Entretanto, ao assumir um enfoque qualitativo defende-se que conhecer o contexto prévio e acadêmico dos sujeitos, as concepções iniciais acerca da disciplina e, ainda, suas estratégias de estudo é mais significativo que analisar os resultados de aprovação, embora um não desconsidere os outros.

Assim, defende-se o enfoque na pesquisa qualitativa, entendendo que a mesma envolve uma grande variedade de materiais empíricos, que podem ser estudos de caso, experiências pessoais, histórias de vida, resultados de interações, enfim, materiais que descrevam a rotina e os significados da vida humana em grupos. Segundo Lefevre, Lefevre e Marques (2009) “A pesquisa qualitativa responde a questões muito particulares. Trabalha com o universo de significados, motivos, aspirações, crenças, valores e atitudes; com um nível de realidade que não pode ser totalmente quantificado" (p. 131).

$\mathrm{O}$ estudo aqui proposto faz parte de uma pesquisa maior, que culminará em uma tese de doutorado. Na tese e, também, no primeiro movimento dessa escrita são considerados os sujeitos da pesquisa os 24 estudantes ingressantes da Casa do Estudante Universitário - CEU, localizadas no campus Rio Grande da FURG, que estão matriculados nas disciplinas Cálculo 
no primeiro semestre de 2017 e ingressaram pelo SISU ${ }^{3}$. Cabe salientar que a FURG está organizada em quatro campi - Rio Grande, São Lourenço do Sul, Santa Vitória do Palmar e Santo Antônio da Patrulha - e, no que tange a moradia estudantil oferece onze CEUs, sendo nove no campus Rio Grande.

Conhecido o contexto de inserção dos sujeitos, o mapa do campo foi dividido em dois movimentos, sendo o primeiro organizado em duas etapas. Esses movimentos sustentam-se nas orientações de Biembengut (2008) acerca do processo de mapear o campo de pesquisa, entendendo que

O mapa de campo conjuga levantamento, organização e classificação de um conjunto de dados, muitas vezes baseado em informações gerais advindas de pessoas ou dados abstratos extraídos de documentos que não retratam totalmente o fenômeno ou questão investigada. Consiste em estabelecer previamente um maior conjunto possível de meios e instrumentos para levantamento, classificação e organização de dados ou informações que sejam pertinentes e suficientes, considerando pontos relevantes ou significativos e que nos valham como mapa para compreender os entes pesquisados (p.101).

Na sequência do texto será descrito como o mapa foi se construindo nas duas etapas no primeiro movimento, com o objetivo de conhecer os sujeitos para os quais os questionamentos seriam feitos no segundo movimento. Contudo, atendendo a proposta de acompanhar os estudantes ingressantes de forma processual, aqui serão apresentados os dados coletados nas entrevistas realizadas com 11 dos 24 sujeitos da pesquisa. Para esses sujeitos foram feitos quatro questionamentos, com o objetivo de compreender as concepções dos estudantes acerca das disciplinas Cálculo, após cursar o primeiro semestre do curso e, ainda, suas estratégias de estudos, a avaliação sobre as mesmas e os fatores que interferem no estudar. Esse artigo se propôs a compreender as concepções acerca da disciplina Cálculo, expressas no primeiro, dos quatro questionamentos.

\section{O primeiro movimento}

O primeiro movimento buscou conhecer o perfil dos 24 ingressantes da moradia estudantil na FURG, para conhecer quem são os sujeitos da pesquisa, no seu contexto que antecede o ingresso na Universidade e, posteriormente traçar o perfil acadêmico desses ingressantes, matriculados nas disciplinas Cálculo, no primeiro semestre de 2017.

\footnotetext{
${ }^{3}$ Sistema de Seleção Unificada - Lei no 12.711, de 29 de agosto de 2012.
} 


\subsection{Conhecendo o perfil dos sujeitos}

Para traçar o perfil dos sujeitos no período que antecede o ingresso na Universidade criou-se uma tabela, com dados coletados no sistema "Acadêmico" da FURG, no qual foram levantados os seguintes dados: procedência regional, gênero, rede de educação básica e idade.

Figura 1: Perfil dos sujeitos pesquisados.

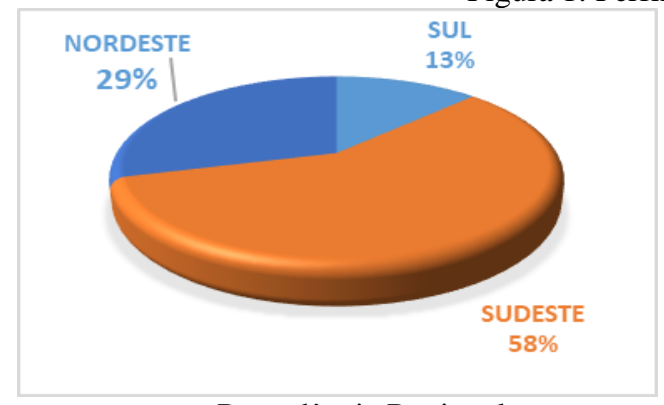

Procedência Regional

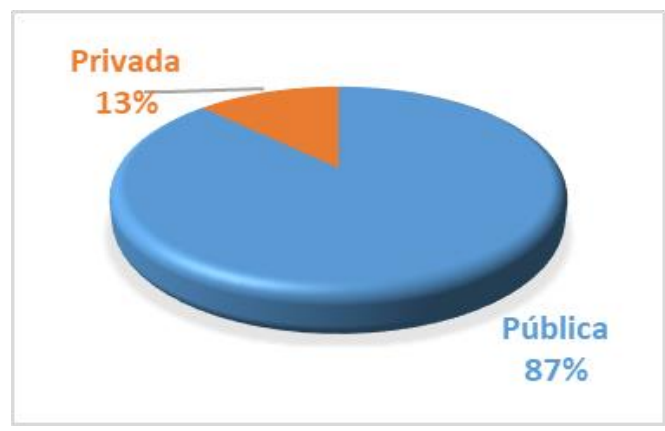

Rede de Educação Básica

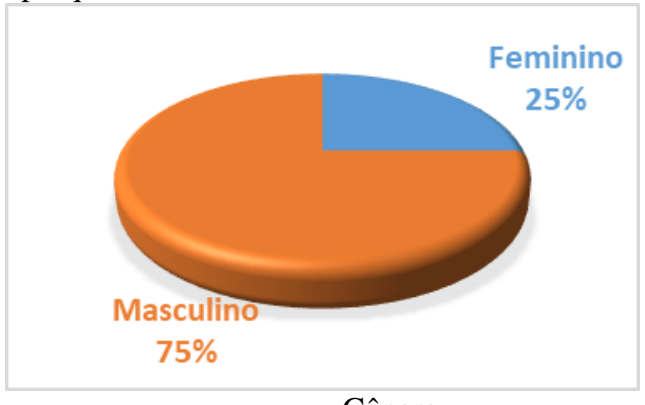

Gênero

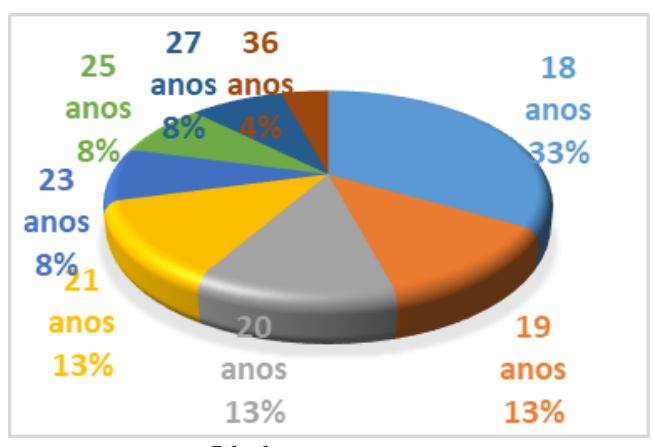

Idade

Fonte: Sistema Acadêmico da FURG.

A Figura 1 apresenta gráficos que evidenciam algumas questões que serão problematizadas com mais profundidade em trabalhos futuros, mas no momento serviram ao objetivo desse estudo, ou seja, conhecer os sujeitos para fazer as primeiras intervenções. Ao observar o gráfico referente a procedência regional identificou-se que 11 (58\%) dos 24 sujeitos da pesquisa são provenientes da região Sudeste e 07 (29\%) são da região Nordeste. Também observa-se um baixo número de estudantes da região Sul, totalizando 03 (13\%), assim como não foram identificados estudantes das regiões Centro-Oeste e Norte.

Em relação ao gênero dos sujeitos, rede de educação básica e idade, o estudo mostrou a predominância de sujeitos egressos da escola pública (87\%), de gênero masculino (75\%) e com idades que variam entre 18 e 21 anos, o último totalizando (72\%).

\subsection{Conhecendo o perfil acadêmico dos ingressantes da moradia estudantil}

Para conhecer o perfil acadêmico considerou-se à modalidade de ingresso pelo SISU, o curso, a disciplina Cálculo em que o estudante está matriculado e as respectivas ementas. 


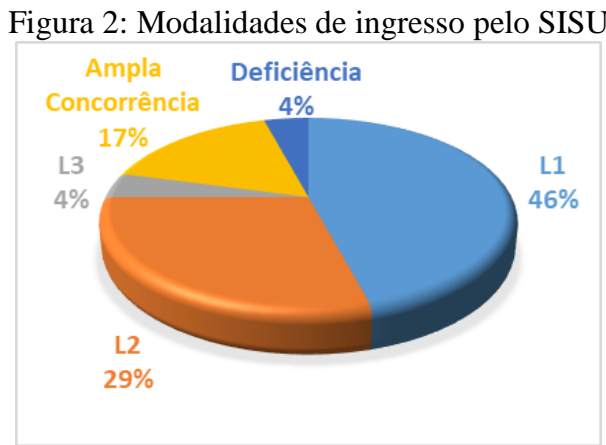

Fonte: Sistema Acadêmico da FURG

Em relação aos dados que buscaram conhecer o perfil dos sujeitos no contexto da Universidade, o gráfico apresentado na Figura 2 evidencia a predominância no ingresso dos sujeitos na modalidade $\mathrm{L}^{4}(46 \%)$ e $\mathrm{L}^{5}$ (29\%) do SISU, que envolvem renda e, tem objetivo de promover o ingresso de estudantes em situação de vulnerabilidade social nos cursos de graduação. E ainda, considerando os ingressantes por ampla concorrência, que totalizam 17\%, os egressos de escola pública representam $83 \%$, reforçando a política do sistema unificado, que tem o objetivo de atender essa demanda. Segundo Daflon, Peres Júnior e Campos (2013)

[...] os alunos egressos de escola pública despontam como os maiores alvos dessas políticas: 60 das 70 universidades com sistemas de cotas, bonificação ou acréscimo de vagas (85\%) visam esse grupo. Em segundo lugar vêm os pretos e pardos (denominados "negros" em alguns programas), em 40 universidades - isto é, 58\% das que tem ações afirmativas. Em terceiro, os indígenas, em $51 \%$ das universidades. Em quarto e quinto, vêm os portadores de deficiência e participantes de programas de formação em licenciatura indígena e, por fim, outros grupos compostos por nativos do estado ou do interior do estado em que a universidade se localiza, professores de rede pública, pessoas de baixa renda, pessoas originárias de comunidade remanescente de quilombos, filhos de agentes públicos mortos ou incapacitados em serviços e mulheres (p. 309-310).

O percentual de sujeitos da pesquisa que ingressaram na modalidade de "pessoa com deficiência", apresentado no gráfico (4\%) vai ao encontro da própria política da FURG, pois até o primeiro semestre de 2017 a Universidade oferecia a modalidade V635, sustentada pelas ações do Programa de Ações Afirmativas - PROAAf, que consiste na reserva de vagas num percentual de 5\% das vagas oferecidas na modalidade de ampla concorrência, por curso/turno, destinada a candidatos com deficiência devidamente comprovada. No $2^{\circ}$ semestre de 2017 , com a mudança da Lei $\mathrm{n}^{\mathrm{o}}$ 12.711/2012, de 29 de agosto de 2012 (Lei de Cotas), que foi

\footnotetext{
${ }^{4}$ L1: Candidatos com renda familiar bruta per capita igual ou inferior a 1,5 salário mínimo que tenham cursado integralmente o ensino médio em escolas públicas (Lei n $\left.{ }^{\circ} 12.711 / 2012\right)$.

${ }^{5}$ L2: Candidatos autodeclarados pretos, pardos ou indígenas, com renda familiar bruta per capita igual ou inferior a 1,5 salário mínimo e que tenham cursado integralmente o ensino médio em escolas públicas (Lei ${ }^{\circ}$ 12.711/2012).
} 
alterada pela Lei $\mathrm{n}^{\circ} 13.409$, de 28 de dezembro de 2016 foram inseridas outras modalidades no SISU, três delas contemplando a reserva de vagas para as pessoas com deficiência.

Em relação aos cursos escolhidos, observa-se predominância pelas engenharias, como mostra a Tabela 1.

Tabela 1: Levantamento do número de estudantes por curso e a disciplina Cálculo cursada em 2017/1:

\begin{tabular}{|c|c|c|c|}
\hline DISC. & PERÍODO & $\begin{array}{l}\mathbf{N}^{\circ} \text { INGRESSANTES POR } \\
\text { CURSO }\end{array}$ & EMENTAS $^{6}$ \\
\hline Cálculo I & Semestral & $\begin{array}{l}02 \text { - Química (Licenciatura) } \\
02 \text { - Química (Bacharelado) } \\
01 \text { - Eng. Química } \\
01 \text { - Eng. de Alimentos } \\
01 \text { - Física (Bacharelado) } \\
01 \text { - Física (Licenciatura) } \\
01 \text { - Matemática Aplicada. }\end{array}$ & $\begin{array}{l}\text { Limites de funções: noção intuitiva, definição, teorema } \\
\text { do confronto, propriedades, limites laterais, limites no } \\
\text { infinito, limites infinitos, indeterminações, limites } \\
\text { fundamentais. Continuidade, teorema de Weierstrass, } \\
\text { teorema do valor médio, tipos de descontinuidade. } \\
\text { Derivadas: motivação, definição, interpretação } \\
\text { geométrica e física, derivabilidade e continuidade, } \\
\text { regras de derivação, derivadas das funções implícitas, } \\
\text { derivadas das funções paramétricas. Propriedades das } \\
\text { funções deriváveis- teorema de Rolle, teorema de } \\
\text { Cauchy, Teorema de L’Hospital. Cálculo de limites } \\
\text { indeterminados. Extremos de funções de uma variável } \\
\text { real: máximos e mínimos, teste da primeira derivada, } \\
\text { teste da segunda derivada. Aplicações. }\end{array}$ \\
\hline Cálculo & Anual & 03 - Oceanologia & $\begin{array}{l}\text { Funções. Conjuntos. Limites funcionais. Continuidade } \\
\text { Derivadas. Diferencial. Máximos e mínimos. Funções } \\
\text { no Rn. Derivadas total e parcial de "n" funções em "n" } \\
\text { variáveis. Máximos e mínimos de funções com duas } \\
\text { variáveis. Diferencial total. Integral indefinida. Integral } \\
\text { definida. Sucessões numéricas e funcionais. Equações } \\
\text { Diferenciais Ordinárias. Equações Diferenciais } \\
\text { Parciais. Gradiente, divergentes e Rotacional. }\end{array}$ \\
\hline $\begin{array}{l}\text { Cálculo } \\
\text { Diferencial } \\
\text { e Integral I }\end{array}$ & Anual & $\begin{array}{l}02 \text { - Eng. Mecânica } \\
01-\text { Eng. Mecânica Empresarial } \\
01-\text { Eng. Civil } \\
01-\text { Eng. Civil Costeira e } \\
\text { Portuária } \\
01-\text { Eng. Civil Empresarial } \\
01-\text { Eng. da Automação } \\
05 \text { - Eng. da Computação }\end{array}$ & $\begin{array}{l}\text { Funções reais de uma variável. Limites. Assíntotas. } \\
\text { Continuidade. Derivadas. Diferencial. Integrais. } \\
\text { Funções no Rn. Diferencial total. Extremos de funções } \\
\text { de duas variáveis. Integração múltipla. Cálculo de áreas } \\
\text { e volumes. }\end{array}$ \\
\hline
\end{tabular}

\section{Fonte: Sistema Acadêmico da FURG}

A Tabela 1 apresenta o número de estudantes por curso, evidenciando que 14 $(58,33 \%)$ dos sujeitos da pesquisa estão matriculados em engenharias, sendo um número expressivo em Engenharia da Computação 05 (20,83\%). Os dados apresentados na tabela mostram também que $09(37,5 \%)$ dos estudantes já concluíram as suas avaliações na disciplina Cálculo I, que é semestral, enquanto 15 (72,5\%) dos sujeitos estão com as disciplinas Cálculo e Cálculo Diferencial e Integral em andamento. Em relação às ementas observa-se, conforme já esperado, significativa semelhança nos conteúdos.

\footnotetext{
${ }^{6}$ Retiradas dos Quadros de Sequência Lógica - QSL dos cursos, disponível: https://www.furg.br/bin/link_servicos/index.php.
} 


\section{O segundo movimento}

Seguindo a perspectiva metodológica de fazer um mapa do campo, o segundo movimento foi fazer a primeira intervenção junto aos sujeitos da pesquisa. Até o momento foram realizadas 11 entrevistas, nas quais elencou-se alguns questionamentos:

\section{1-Defina a disciplina Cálculo em uma palavra;}

02-Como você estuda Cálculo?

03-Como você avalia a sua prática de estudo em relação ao objetivo de aprender e aprovar em Cálculo?

04-Na sua avaliação, que outros fatores, além das práticas de estudos podem influenciar na sua aprendizagem em Cálculo?

Realizadas as primeiras intervenções, optou-se por fazer um recorte e apresentar neste texto algumas expressões que definem as disciplinas Cálculo, segundo os sujeitos entrevistados (questionamento 1). Esse recorte indicou uma possibilidade de compreender as concepções dos estudantes acerca das disciplinas Cálculo, após cursar o primeiro semestre do curso. As expressões que os estudantes utilizaram para definir a disciplina Cálculo foram: divisor de águas, complicado (02), paixão, medo, difícil (02), possibilidades, empecilho, necessário (02). Foi possível observar que os estudantes das engenharias utilizam palavras como paixão, necessário e possibilidades para definir o Cálculo. Essas expressões vão ao encontro da proposta de formação que os estudantes buscam e suas expectativas em relação as disciplinas do curso e os conhecimentos necessários para torna-se engenheiro. Para Alves et al (2016)

\footnotetext{
Independentemente da área de estudo, os conceitos matemáticos são essenciais na formação de engenheiros, quer na compreensão dos diferentes conceitos, quer no conhecimento específico da sua aplicabilidade. Durante um curso de Engenharia, os estudantes aprendem e consolidam os princípios básicos de Matemática para resolver problemas práticos, reforçando o conhecimento de conceitos matemáticos nomeadamente das áreas de estatística, métodos numéricos, otimização e simulação, entre muitos outros (p. 260).
}

Assim, as especificidades dos cursos de engenharias estão expressas na própria concepção dos sujeitos sobre as disciplinas Cálculo, pois mesmo que enfrentem dificuldades de aprendizagem, a escolha profissional está condicionada a aprendizagem de conceitos da matemática avançada (ALMEIDA e IGLIORI, 2013). Entretanto, os estudantes matriculados nas Engenharias, Matemática, Física e Química mostraram-se bastante motivados para 
aprender Cálculo, manifestado em expressões como: divisor de águas, complicado e difícil. Para Alves et al (2016)

A Matemática tem uma multiplicidade de atributos que vão desde o desenvolvimento do raciocínio lógico de uma pessoa até à compreensão de estruturas abstratas. Ela promove o pensamento lógico e racional e aumenta a capacidade de analisar e resolver problemas. Os conceitos matemáticos surgem no currículo de muitos cursos, incluindo cursos de Engenharia, e revela-se essencial para a formação de todos os futuros engenheiros, independentemente da área de estudo e de trabalho (p.261).

Uma outra natureza de concepção foi evidenciada na definição expressa por dois sujeitos matriculados no curso de Oceanologia, são elas: medo e empecilho. Diferente dos sujeitos matriculados nos cursos de Engenharia, Matemática, Física e Química, não existe argumentos explícitos ou implícitos acerca da necessidade de aprender Cálculo, ou conceitos de matemática avançada (ALMEIDA e IGLIORI, 2013) e, ainda, as expressões apresentadas não sugerem nenhum tipo de motivação para tal aprendizagem. Para Alves et al (2016)

\begin{abstract}
A motivação dos estudantes para a aprendizagem de conceitos matemáticos é uma das principais preocupações dos educadores e dos investigadores em educação. A motivação é a força motriz que está por detrás das nossas ações e que condiciona as nossas necessidades, desejos e ambições na vida. Estudos mostram existir uma relação entre a motivação para a obtenção de resultados e o desempenho académico de estudantes, no ensino superior (Bakar et al., 2010; Sirmaci, 2010; Walter \& Hart, 2009) (p. 264).
\end{abstract}

Dito isso, ainda observa-se que em geral os estudantes que escolhem a Oceanologia como profissão tem bastante afinidade com a área de Ciências Biológicas. Entende-se assim que os aspectos que influenciam o desempenho na disciplina Cálculo podem ir além do nível da dificuldade de aprendizagem, mas estar relacionados a própria concepção evidenciada nas expressões - medo e empecilho - uma disciplina na qual muitas vezes o estudante não se sente seguro e, ainda, apresenta-se como um grande obstáculo na sua formação.

\title{
5. Considerações Finais
}

Conclui-se o presente artigo defendendo que essa pesquisa se mostrou relevante para os sujeitos envolvidos, pesquisadoras e a comunidade acadêmica como um todo, visto que a mesma contribui para qualificar as ações do acompanhamento pedagógico das disciplinas Cálculo. De forma mais imediata, entende-se que a construção do mapa do campo, como movimento inicial de contextualização e intervenção com os sujeitos de pesquisa produziu conhecimentos significativos tanto na área do Cálculo, especificamente das estratégias de 
estudo e aprendizagem dos conteúdos relacionados, como acerca das questões que envolvem a políticas de ingresso e permanência promovido pelo SISU, nas quais inserem-se estudantes ingressantes da moradia estudantil, sujeitos dessa pesquisa.

Em relação as expressões que os estudantes utilizaram para definir as disciplinas Cálculo - divisor de águas, complicado, paixão, medo, difícil, possibilidades, empecilho, necessário - entende-se que as mesmas evidenciam concepções que vão se construindo muito relacionadas as áreas que os sujeitos buscam formação. Os estudantes da Engenharia, Matemática, Física e Química mostram-se motivados a aprender Cálculo, mesmo que as dificuldades lhes imponham alguns limites, pois é consenso a sua importância na formação desses sujeitos. No entanto, os estudantes matriculados em Oceanologia apresentam uma outra natureza de concepção, pois as expressões manifestam um sentimento de medo e limitações, que possivelmente não estão relacionadas apenas as dificuldades de aprendizagem, mas a própria motivação para o aprender.

\section{Referências}

ALARCÃO, I. (2000). Para uma conceptualização dos fenómenos de insucesso/sucesso escolares no ensino superior. In J. Tavares \& R. Santiago (Ed.), Ensino superior: (In) sucesso académico (pp. 13-23). Porto: Porto Editora.

ALMEIDA, M. V. de; IGLIORI, Sonia Barbosa Camargo. Educação Matemática no Ensino Superior e abordagens de Tall sobre o ensino/aprendizagem do Cálculo. Educação

Matemática Pesquisa São Paulo, v.15, n.3, pp.718-734, 2013.

ALVES, M; COUTINHO, C; Rocha, A. M; RODRIGUES, C. Fatores que influenciam a aprendizagem de conceitos matemáticos em cursos de engenharia: Um estudo exploratório com estudantes da Universidade do Minho. In: Revista Portuguesa da Educação, n. 29, pp 259-293, 2016.

BIEMBENGUT, M. S. Mapeamento na pesquisa educacional. Rio de Janeiro: Ciência Moderna, 2008.

BRASIL. Lei $\mathrm{N}^{\circ}$ 12.711, de 29 de agosto de 2012. Dispõe sobre o ingresso nas universidades federais e nas instituições federais de ensino técnico de nível médio e dá outras providências. Disponível em http://www.planalto.gov.br/ccivil_03/_ato2011-2014/2012/lei/112711.htm. Acesso em 16 out. 2017.

BRASIL. Lei $\mathrm{n}^{\mathbf{0}}$ 13.409, de 28 de dezembro de 2016. Altera a Lei $\mathrm{n}^{\mathrm{o}} 12.711$, de 29 de agosto de 2012, para dispor sobre a reserva de vagas para pessoas com deficiência nos cursos técnico de nível médio e superior das instituições federais de ensino. Disponível em http://www.planalto.gov.br/ccivil_03/_ato2015-2018/2016/lei/L13409.htm. Acessado em 16 out. 2017. 
DAFLOM, V. T; PERES JÚNIOR, J; CAMPOS, L. A. Ações Afirmativas Raciais no Ensino Superior Público Brasileiro: Um Panorama Analítico. In.: Cadernos de Pesquisa, v. 43, n.148, p. 302-327, jan/abr 2013.

GARRIDO, E. N.; MERCURI, E. N. G. da S. A moradia estudantil universitária como tema na produção científica nacional. In.: Revista Semestral da Associação Brasileira de Psicologia Escolar e Educacional, SP. v. 17, n. 1, Janeiro/Junho de 2013: 87-95

LEFEVRE F.; LEFEVRE, A. M. C; MARQUES, M.C.C. Discurso do Sujeito Coletivo, complexidade e auto-organização. In.: Ciências e Saúde Coletiva. 2009; 14(4):1193-1204.

MALTA, I. Linguagem, leitura e matemática. In: CURY, H. N. Disciplinas matemáticas em cursos superiores: reflexões, relatos, propostas. Porto Alegre: EDIPUCRS, 2004. p.41-62.

SCHIRMER, S. N; BURLAMAQUI, L. do A; MAURELL, J. R. P. Programa De

Acompanhamento e Apoio Pedagógico ao Estudante: vivências e experiências no âmbito das ações afirmativas na FURG. In.: A política de Ações Afirmativas na FURG: um espaço de Formação Permanente. PEREIRA, Vilmar Alves; SCHIRMER, Sirlei Nádia; JARDIM, Daniele Barros (Org.). Rio Grande: Editora da FURG, 2016. p. 147 - 156.

SILVA, B. A. da. Diferentes dimensões do ensino e aprendizagem do Cálculo In.: Educ. Matem. Pesq., São Paulo, v.13, n.3, pp.393-413, 2011. 